\title{
Healing Health Care: From Sick Care Towards Salutogenic Healing Systems
}

\author{
Christopher J. Fries ${ }^{1}$
}

Published online: 11 April 2019

๑) Springer Nature Limited 2019

\begin{abstract}
Aaron Antonovsky's salutogenic model is starting to impact health promotion at the level of theory and research. However, the benefits of Antonovsky's theory for population health promotion practices and health care restructuring are unrealized. This analysis uses the definition of health derived from complexity science as a lifelong, multidimensional adaptive process comprised of intersecting biological, psychological, social, environmental, and spiritual systems as a starting point for a salutogenic analysis of formal healthcare. Following Antonovsky's criticism of contemporary healthcare as resting upon a pathogenic paradigm, I outline four general shortcomings associated with the pathogenic approach to healthcare. The basic elements of a healthcare system designed according to principles derived from Antonovsky's salutogenic model of health are then presented. It is argued that Antonovsky's theory offers a productive basis for conceptualizing health and healthcare systems in that it allows us to grasp that debates between population health promotion and providing medical care, are, at their root, unproductive debates predicated on a false dichotomy. A salutogenic healthcare system is one which pays credence to the nested complexity of human health and strives to strike an adaptive balance between health production and the provision of medical care.
\end{abstract}

Keywords Health sociology $\cdot$ Salutogenesis $\cdot$ Health care policy

\section{Introduction}

Health and wellness cannot be fully understood by studying sickness. This is the main idea behind the salutogenic model of health developed by Aaron Antonovsky (1923-1994) (1979, 1987). To understand the origins of positive health, Antonovsky sought to understand the salutary (i.e., health promoting) factors related to the

Christopher J. Fries

Christopher.Fries@umanitoba.ca

1 Department of Sociology and Criminology, University of Manitoba, 317 Isbister Building,

Winnipeg, MB R3T 2N2, Canada 
production of health and wellness. He was a medical sociologist who became interested in studying health, rather than sickness; the preoccupation of so many of his colleagues. Highlighting the importance of adopting a salutogenic model, Antonovsky (1979, p. 12) declared, 'The problem of salutogenesis is one of the most mysterious, intriguing, and meaningful challenges for philosophy and the biological and the social sciences'. For Antonovsky (1979, 1987, 1996), given the stressful and hazardous conditions that surround people as part of daily life, the origins of health and how anyone manages to stay well are truly a mystery. Describing Antonovsky's salutogenesis, Tones and Green (2004, p. 10) explain, 'Central to this theory is the challenge posed by the complexities and uncertainties of the world'. In his own words, 'Given the ubiquity of pathogens_-microbiological, chemical, physical, psychological, social, and cultural-it seems to me self-evident that everyone should succumb to this bombardment and constantly be dying' (Antonovsky 1979, p. 13). Antonovsky's insight was that despite the pathogenic onslaught of all these factors that threaten health, remarkably, most people are able to remain well and live happy and productive lives; most of the time. The important question for Antonovsky was how this was possible. As Eriksson and Lindstrom (2008, p. 191) explain the rationale behind Antonovsky's research, 'He was intrigued by the question why some people, regardless of major stressful situations and severe hardships, stay healthy while others do not'. It was this mystery that Antonovsky sought to unravel with his concept of salutogenesis, referring to the origins of health and wellness. He placed the concept of salutogenesis at the heart of his efforts to unravel the mysterious origins of health and wellness.

Since Antonovsky first introduced his salutogenic model in 1979 a growing number of researchers have adopted salutogenesis as a conceptual basis for their studies. Versions of the Sense of Coherence (SOC) scale he developed to research the psychosocial origins of health have been used in several large-scale population health surveys across the globe (Eriksson and Mittelmark 2017). In 2007, the International Union for Health Promotion and Education formed a Global Working Group on Salutogenesis. There is a Center on Salutogenesis at University West in Sweden. A search of the multi-disciplinary abstract and citation database Scopus using the terms 'salutogenesis' and 'Antonovsky' returns 432 studies published since 1990. 2017 witnessed the publication of The Handbook of Salutogenesis, containing contributions from salutogenic scholars from around the world. These are positive developments, which have contributed greatly to the growing scholarship on salutogenesis and understanding the mysterious origins of health. However, while the salutogenic model of health is starting to impact health promotion at the level of theory and research, the benefits of Antonovsky's formulation for population health promotion practices and healthcare restructuring are, at present, unrealized with the pathogenic paradigm still dominant in considerations of health, healing, and healthcare (Friedli 2013).

In this paper, I set the salutogenic model as a conceptual guide to reforming the formal healthcare system. As Pelikan (2017, p. 263) argues, 'salutogenic thinking has good potential to be applied to health care in relation to health promoting interventions for the health of patients, staff, and citizens, and in supporting health promoting structures and cultures of health care institutions for better everyday practice 
and policy'. While Antonovsky (1979) suggested as much, unfortunately he never fully developed this line of reasoning before his death at the age of 71 . This paper is offered as an initial step in applying the salutogenic model to healthcare reform. It sets out what can accurately be read as a utopian vision for the reform of formal healthcare in line with Antonovsky's salutogenic model. I begin by following Antonovsky to critically outline the features of contemporary healthcare originating within a pathogenic paradigm, highlighting key shortcomings of this approach. The analysis then outlines the basic elements of a healthcare system designed according to principles derived from Antonovsky's theory. I conclude by suggesting opportunities for healing healthcare systems in line with principles derived from the salutogenic model of health in light of existing political, economic, and sociocultural factors that stand as barriers that must be overcome to achieve the vision of healing healthcare.

\section{The Pathogenic Paradigm of Contemporary Healthcare}

Despite what would seem to be the obvious importance of understanding salutogenesis, medical and health researchers continue to be preoccupied with pathogenesis, which Antonovsky (1996, p. 13) argued, 'suffuses all western medical thinking'. As Lindström and Eriksson (2010, p. 20) describe, 'In general there is much more knowledge and information on what causes disease and the treatment of these conditions (the pathogenic orientation) than on what causes and then maintains and develops good health (the salutogenic orientation)'. The term 'pathogenesis' is derived from the Greek terms 'pathos', meaning disease, and 'genesis', meaning origins. 'Pathogens' are disease causing agents (e.g., germs and viruses). Thus, the pathogenic paradigm represents an approach to understanding health and illness that is focused on understanding the origins of and treating disease. Becker and colleagues (2010, p. 2) note, 'Pathogenesis starts by considering disease and infirmity and then works retrospectively to determine how individuals can avoid, manage, and/or eliminate that disease'. Antonovsky (1987, p. xii) explains, 'A pathological orientation seeks to explain why people get sick, why they enter a given disease category'. The emphasis of the pathogenic approach is on understanding the causes and consequences of disease, rather than understanding the factors that promote health and healing. The limiting idea behind the pathogenic paradigm is that by studying sickness we can come to understand and enhance health. In fact, in this approach health is defined merely as the absence of disease.

The contemporary formal healthcare system is structured upon the pathogenic paradigm. Antonovsky (1979) was one of the first to criticize the widespread pathogenic orientation of healthcare, which he termed the disease care system, owing to its focus on sickness rather than understanding the factors that promote health and healing. Many researchers, such as Jonas and colleagues, have followed Antonovsky to argue that 'Medicine teaches and organizes its activities from research to reimbursement on pathogenesis' (2014, p. 86). Both clinical medicine and medical researchers remain, for the most part, preoccupied with disease, rather than understanding the factors that contribute to health and healing. As a revolutionary alternative to the 
pathogenic paradigm, Antonovsky developed a model of human health $(1979,1987$, 1996) centered not around pathogenesis, but rather on 'salutogenesis'; the origins of health.

The revolutionary idea advanced by Antonovsky was that traditional diseasebased approaches focusing on understanding and treating illness are not the most effective means of producing a healthy population. Nor is the pathogenic paradigm the most effective basis for a healthcare system. The realization that the so-called 'healthcare system' is, put bluntly, more accurately described as a 'disease care system' or what can be termed 'sick care', motivated Antonovsky to search for an alternative approach.

As far back as 1972, Winkelstein criticized the pathogenic orientation of traditional healthcare observing that 'our society's clinical practice and scientific research, focuses on and responds to a particular disease or clinical entity' (as cited in Antonovsky 1979, p. 15). More recently, Eriksson and Lindstrom (2008, pp. 190-191) state, 'the biomedical or pathogenic approach where health is generated through the elimination of risks for diseases is the dominating paradigm at present'. The biomedical model that informs contemporary health care stresses the importance of understanding the etiology (origins) of disease and the nature of the pathology (sickness) (Segall and Fries 2017). As Segall and Fries (2017, p. 320) explain, the biomedical model is 'a model of healthcare based upon scientific understanding that, at the level of basic knowledge, understands health and illness in terms of biological processes and, at the applied clinical level, privileges individualized, biologically orientated, pharmacological, surgical, and technological interventions'. The biomedical model rests on a pathogenic paradigm. The biomedical care system's preoccupation with discovering the biological causes of disease is motivated by the taken-for-granted belief that this will lead to the discovery of effective means of treatment and eventually result in biomedically based cures (i.e., pharmaceutical drug therapies and surgical or other technologically based treatments). While these are commendable goals, the shortcomings of such a medicalized approach to healthcare based upon the pathogenic paradigm are becoming obvious. The following section outlines four general shortcomings associated with the pathogenic approach to healthcare.

\section{Shortcomings of Sick Care}

\section{Individualization of Health}

Antonovsky explains that 'the disease care institution, organized around the problem of pathology, is more comfortable with the individual patient who has come down with a diagnosable disease for which effective therapy is available' (Antonovsky 1979 , p. 45). One consequence of this is that 'health' becomes individualized. That is, health, reduced to the absence of disease, is understood from the perspective of individuals rather than groups or the environment. As Antonovsky (1987, p. 4 sic) explains, 'attention is given to the pathology, not the human being who has a particular problem... to be blind to the sickness of the person, to his total life situation, 
to his suffering, is not only inhumane; it leads to a failure to understand the etiology of the person's state of health'. The mechanistic approach of sick care directs focus to individual biology and behaviours. Health is understood as the property of individuals who are held individually responsible for their health status (Lupton 1995). Medical interventions are then targeted at individuals and individual behaviour; removed from its systemic context (Holman et al. 2018). Antonovsky (1979, p. 37, 66) describes this as the "'magic bullet" approach-one disease, one cure... pathogenesis... pushes in the direction of the single disease and the single bullet'. This individualization of health aligns well with the prevailing neoliberal political ideology in most advanced western societies in that it puts the emphasis on the individual (Ayo 2012; Fries 2008; Schrecker and Bambra 2015). The drawback with this is that health cannot correctly be reduced to matters of individual biology or behaviour alone. As Antonovsky (1996, p. 14) argues, 'It is, I believe, impermissible to identify a rich, complex human being with a particular pathology, disability or characteristic. I submit that, working with a pathogenic orientation, one is pushed in this direction, pressured to forget the complexity'. This approach fails to appreciate the complexity of human health and wellness. From the perspective of complexity science (Geyer and Rihani 2010) health is a lifelong, multidimensional adaptive process comprised of intersecting biological, psychological, social, environmental, and spiritual systems. However, under the influence of the pathogenic paradigm, sick care reduces health to a matter of individualized biology and behaviour.

\section{Exclusion of the Non-diseased}

A related problem with the pathogenic paradigm is, as Antonovsky (1979) argues, that such an approach to health care excludes those who are non-diseased, who amount to about two-thirds of the general population. The capacity of people to manage their own health; 'self-care', is overlooked as an important population health promotion mechanism. This drawback becomes apparent when examining the total health expenditures by type of service. The OECD (2017) notes that in 2015 member countries devoted only six per cent of their health spending towards prevention and public health services. By definition, public health involves services directed towards the health of the entire population or specific subpopulations, and includes measures such as: vaccination programs; food, drug, occupational, and environmental safety inspections; community mental health programs; epidemiological surveillance and medical screening; population health assessment; health education and promotion; and disaster response (CIHI 2015; Naylor 2003).

In contrast, almost two-thirds of health spending was on curative, rehabilitative, and long-term care; sick care (OECD 2017). When you add in the 19 per cent spent on medical goods such as pharmaceuticals, this proportion rises to over 80 percent. By a conservative estimate, over three-quarters of total healthcare expenditures have a pathogenic focus. As the OECD data make clear, the contemporary sick care systems of developed nations are overwhelmingly focused on providing personal healthcare services rather than public health. 
Obscured in these expenditure data is that public health practices, for the most part, as defined by the OECD have a disease prevention, rather than a health promotion focus. A report prepared for the Public Health Agency of Canada in the wake of the SARS outbreak in 2003 makes the important point that, 'Tellingly, reliable information on expenditures on public health... is not even readily available' (Naylor 2003, p. 54). Naylor et al. point out that much of what is frequently termed 'public health' includes factors not directly related to public health, such as the bureaucracy surrounding formal healthcare, and that researchers have no real way of measuring how much of spending is actually directed at population health. The report's authors go on to estimate that, adopting a broad definition, no more than three percent of total health expenditures in Canada are devoted to public health. They conclude, 'Notwithstanding the drumbeat of disease prevention and health promotion, governments have steadily committed virtually all new health spending to areas other than public health' (2003, p. 55). In general, advanced healthcare systems talk a good game about public health while in reality, little is done to promote public health.

A paradigm shift has not yet occurred from pathogenesis to salutogenesis which would allow researchers to measure how many resources are truly being invested not just in disease prevention but health creation. What is known for certain is that the majority of formal healthcare services are designed to provide illness intervention and treatment once disease has already occurred. The focus of sick care remains on individual health needs in terms of clinical risk factors and disease management. This leads Segall and Fries (2017, p. 119) to conclude, 'At best, formal health services have a (personal) disease-avoidance focus rather than a (population) health maintenance emphasis'.

'At the core of the pathogenic paradigm, in theory and in action, is a dichotomous classification of persons as being diseased or healthy' (Antonovsky 1979, p. 39). These limiting definitions of health are frequently employed in pathogenic medical research because of their simplicity (Millar and Hull 1997). It is as easy as it is misleading to study health using quantitative indictors, such as mortality rates, survival periods after diagnosis, incidence or prevalence of diseases or risk factors (Evans and Stoddart 1990). Health services research, guided by the pathogenic paradigm, defines health by what it is not; sickness, and medicocentric researchers attempt to understand health by studying disease. The nested complexity of population health (Henly et al. 2011), is ignored by the pathogenic paradigm.

\section{Retroactive Response to Illness}

The pathogenic paradigm is driven by the taken-for-granted belief that by focusing on illness and disease medical researchers come to understand these phenomena better, and hopefully come up with ways of relieving suffering once it occurs. However, looking at health through the lens of sickness fails to provide insight into the origins of health or instruct how to create health and promote healing in the first place. Rather, the focus of sick care is on responding reactively to illness once it has occurred. Much less effort is directed at proactively understanding and producing personal and population health. For instance, health researchers have been unable 
to determine to what extent medicine and medical knowledge actually contribute to improved population health (Markle and McCrea 2008). In terms of the traditional model of medical care, what ends up happening is investing money and research effort in designing the formal health care system as a sort of 'repair shop' (Kickbusch 2007), rather than a system intended to create and foster health. The pathogenic paradigm results not in 'health' care, but rather what we end up with is 'sick' care; retroactive response to disease.

\section{Promotion of Medicalization and Medical Dominance}

The historical development of the sick care system has resulted in a medicalized system focused on the acute care of the already sick within costly and high-tech institutions, such as hospitals, under the professional control of physicians (Segall and Fries 2017). Sociologists use the term medicalization to describe the process whereby aspects of life come to be defined as medical issues requiring intervention and control on the part of professional medicine (Ballard and Elston 2005; Busfield 2017; Conrad 2007; Segall and Fries 2017). Medicalizing phenomena involves reducing them to biophysical causes and prescribing biomedically focused responses. Because medical professionals are viewed as having expertise in dealing with biomedical issues, physicians hold contested responsibility for defining and managing aspects of life that have been medicalized (Van den Bogaert et al. 2017). The list of phenomena that have been medicalized is long and varied (Ballard and Elston 2005; Busfield 2017; Conrad 2007). In the 1970s Eliot Freidson (1970a, b) famously coined the term medical dominance to describe the political process whereby physicians act as an organized professional group upon which the government confers the powers of self-regulation and professional autonomy in setting standards of medical practice and care. In this way, medicalization renders more and more aspects of life under the control and influence of the medical profession and expands medical dominance. The sociology of medicine has long since established that medicalization individualizes phenomena through focusing on biophysical factors, discursively framing causes and treatment in pathological terms, while directing attention away from social and environmental factors towards medical management and control (Zola 1972). In this way the pathogenic paradigm of sick care promotes medicalization and medical dominance.

Disease care is obviously necessary and invaluable. However, the sick care system was not structured to meet the major health challenges facing advanced countries today (Breslow 2004). Contemporary formal healthcare emerged primarily in response to infectious disease while the main problem now is chronic illness, linked to lifestyle and environmental factors. Breslow (1972) describes the shift from epidemic infectious disease to chronic illness as representing 'an essentially new kind of health problem' (as cited in Antonovsky 1979, p. 54). He later (2004) describes the epidemiologic transition to chronic disease as the major health problem as representing a second revolution in health. Breslow explains that, 'changes in conditions of life and behavior, together with longer lives lived, during which bodily damage can accumulate, have resulted in the modern chronic disease epidemics, including 
heart disease, cancer, stroke, and diabetes'. This revolution is not complete in that while medicine may be becoming better equipped to understand and manage such chronic illnesses, such conditions still make up the majority of the burden of morbidity and mortality. He argues that, "The current situation now calls for a third health revolution on the basis of a view of health as a "resource for everyday life", "not merely the absence of disease and infirmity"'. Referring to the World Health Organization's 1986 definition of health, he explains that the successes of biomedical healthcare perpetuated higher public expectations with regard to health and healing. In part a victim of its own success, increasing medicalization has meant that public expectations of professional medicine are higher than ever. No longer content with minimizing suffering and disease, people now desire and indeed, expect, lifelong wellness. To meet these challenges the disease care system has to remake itself into a salutogenic, healing healthcare system. Next, I outline the basic elements of a healthcare system designed according to principles derived from Antonovsky's salutogenic model.

\section{Elements of a Salutogenic Healthcare System}

While the sick care system is oriented toward reacting to disease once it has occurred, a salutogenic system is a more ambitious one which extends beyond treatment of illness and injury; oriented not only toward the prevention of illness, but contributing to the production of both individual and population health and wellness. It moves from the deficit-based model to one which is assets based (Friedli 2013; Morgan et al. 2010). Reflecting the pathogenic paradigm's influence, most discussions of healthcare systems are limited in that they are predicated on 'a fundamental dichotomy between healthy and sick people' (Antonovsky 1987, p. 3). As Antonovsky (1979, p. 5) points out, however, the healthy/sick dichotomy is a false dichotomy in that 'all of us, as long as we are alive, are in part healthy and in part sick'. As depicted in Fig. 1, Antonovsky adopted a continuum model of health, similar to Rose's (2008) famous public health application of the continuous distribution, which rather than viewing people dualistically as either sick or healthy, views health as a relational process with people continuously moving between good health (wellness) and sickness. 'Health is here seen as a movement or a process, where people are always in some respects healthy and independent of existing distress and diseases' (Lindström and Eriksson 2010, p. 32). In this model, sickness and wellness

\section{The Continuum Model of Health}

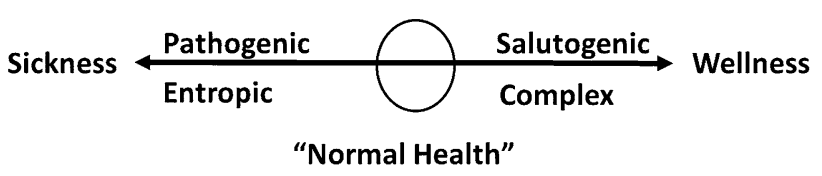

Fig. 1 Antonovsky's continuum model of health 
are not opposing forces, but rather interrelated dimensions of human experience. So-called 'normal health' involves continuous movement along the healthy/disease) continuum. '[A]ll of us, as long as we are alive, are in part healthy and in part sick... (Antonovsky 1979, p. 5). Such an understanding of health stands in stark contrast with pathogenic definitions of health which are limited in that they define health, negatively, as the absence of disease. 'Salutogenesis asks, What are the factors pushing this person toward this end or toward that end of the continuum?' (Antonovsky 1979, p. 37). To unravel this mystery, Antonovsky developed and empirically elaborated two interrelated key concepts of the salutogenic model; the sense of coherence and generalized resistance resources. I will now briefly review each of these before presenting them as basis for reforming the formal healthcare system along salutogenic lines.

\section{The Sense of Coherence and Generalized Resistance Resources (GRRs)}

Antonovsky's quest to understand the origins of health focused on an attitudinal disposition he described as the sense of coherence (SOC). The SOC is 'a way of looking at the world' (Antonovsky 1979, p. 8) and coping with stressors that sees life as understandable, manageable, and meaningful. According to Antonovsky (1987, p. $15)$, 'the sense of coherence is a very major determinant of maintaining one's position on the health ease/dis-ease continuum and of movement towards the healthy end'. Antonovsky describes the SOC as 'a global orientation that expresses the extent to which one has a pervasive, enduring though dynamic feeling of confidence that one's internal and external environments are predictable and that there is a high probability that things will work out as well as can reasonably be expected' (1979, p. 123). The SOC can be understood as embedded with what Bourdieu (1977) famously terms the habitus. It is an embodied disposition for effective coping that rests upon early socialization experiences, and though relatively stable, once formed, is subject to modification given changing social conditions. Those with SOC are highly resilient and adaptable in the face of life's obstacles. It is not that such people 'walk between the raindrops', as Antonovsky metaphorically puts it (1979, p. 77). We all face hardships, have to deal with stressors, and become ill at points during the life course. What sets those with SOC apart is the positive and productive attitude with which they understand and meet these challenges.

Antonovsky (1979) suggested that the origins of the SOC and of health lay with what he termed, 'generalized resistance resources'. As he explains, 'From the time of birth, or even earlier, we constantly go through situations of challenge and response, stress, tension, and resolution. The more these experiences are characterized by consistency, participation in shaping outcome, and underload-overload balance of stimuli, the more we begin to see the world as being coherent and predictable' (1979, p. 187). Generalized resistance resources (GRRs) are, as the name implies, resources that can be used to avoid, or reduce, or both, the harm caused by stressful life events, which is potentially damaging to health. For Antonovsky, a GRR is 'any characteristic of the person, the group, or the environment that can facilitate effective tension management' (1979, p. 99). In keeping with his interests 
in stress and coping, they are resources for dealing with stressors. GRRs run the range from genetic and biophysical properties of the individual all the way up to the structural and cultural properties of societies. As such, they operate across the micro and macro levels of sociological analysis. GRRs are an expansive concept, including factors such as: adaptability on the physiological, biochemical, psychological, cultural, and social levels; profound ties to concrete, immediate others; and commitment of and institutionalized ties between the individual and the total community (Antonovsky 1979, p. 100).

Antonovsky (1979) explains, GRRs may be the property of individuals, such as genetic endowment, intelligence, and particular health behaviours (such as balanced diet or engaging in regular physical exercise), or collectivities, such as social cohesion and social support networks. GRRs are tremendously important for health in that, 'Whatever one's location at a given point in time on the health ease/dis-ease continuum, the extent to which GRRs are available to one plays a decisive role in determining movement toward the healthy end of the continuum or, at least, holding one's own' (Antonovsky 1979, p. 100). Successful deployment of GGRs to cope with life's challenges builds up and reinforces the SOC. In this, early childhood experiences are decisive. Thus, according to the salutogenic model, the origins of health are to be found in GRRs and the SOC.

The SOC is formed in relation to the availability and efficacy of GRRs (Antonovsky 1979). As Alivia et al. (2011, p. 382) explain the SOC is a property that rests upon socialization experiences and develops across the lifecourse: 'Although SOC develops naturally in the first 30 years of life it is not a static orientation. It can be strengthened through personal activity and care'. Societies that foster GRRs are healthy societies in that they provide members with the resources needed to develop a strong SOC. GRRs can also be the property of collectivities, such as groups and organizations (Antonovsky 1987). However, throughout society, the distribution of GRRs is like the distribution of health, unequal. In fact, according to Antonovsky (1979) inequities in GRRs account for observed disparities in health. The key point is that it would potentially be extremely salutogenic if physicians and the formal health care system could be configured so as to function as GRRs that enhance the SOC. In terms of the socially constructed and culturally contested nature of healthcare, Antonovsky was however, acutely aware of the dangers of medicalization, warning, 'The sense of coherence... is hardly strengthened by having the health institution responsible for all aspects of peoples' lives' (1979, p. 53). Guided by his salutogenic model, this analysis concludes by suggesting opportunities for healing the health care system in line with salutogenic principles.

\section{Principles of a Salutogenic Healthcare System}

\section{Recognize Health as a Complex Adaptive Process}

In keeping with defining health is a lifelong, multidimensional adaptive process, Renaud (1994, p. 322) explains that health is all about adapting positively to changing life circumstances: 
... health derives in part from our ability to adapt and the faith in the future that we develop as children, as well as from the friendship and support networks to which we have access at work, at home, and in the community. Health also stems from our sense of having room to manoeuvre along with some control over our work, and from our capacity for dealing with abrupt changes in our lives (unemployment, separation, death, etc.).

In this way, health is a complex social capacity, shaped and influenced by systemic interactions occurring across the life course. The goal of a salutogenic health care system then, 'In contradistinction to the search for magic-bullet solutions' is to 'facilitate active adaptation... to the environment' (Antonovsky 1987, p. 3). From the perspective of complexity science, health is about adaptability, therefore, it follows that a salutogenic health care system must facilitate adaptability. For Alivia et al. (2011, p. 381) this means 'The choice of medical intervention needs to take into account the technological advances of biomedicine but tailor them to the physical, psychological and spiritual needs of the patient in the context of their biography.' The primary directive for a salutogenic health care system must be '...enhancing the adaptive capacity of human beings' (Antonovsky 1979, p. viii). This is achieved in relation to the SOC.

\section{Sustain, Restore, and Enhance the SOC}

Given that the SOC is the centerpiece of the salutogenic model, it is of obvious importance that healing systems be structured around the sustenance, restoration, and enhancement of the SOC. While Antonovsky initially dismisses the idea that formal care can significantly affect the SOC as 'utopian', he did concede that, 'One way, then, for the clinician to modify the SOC — or at least not modify it negativelyis to structure encounters so that this damage is not done' (1987, pp. 124-125). As Alivia et al. explain (2011, p. 384) this means that 'Medical interventions should not only be aimed at removing the disease but also at improving health by strengthening a person's SOC and their resilience at physical, psychological and spiritual levels'. While many clinical encounters within the sick care system are iatrogenic (Illich 1976), a healing healthcare system is one in which patients are supported in efforts to understand their healthcare experiences as orderly, manageable, and meaningful. As Pelikan (2017, p. 263) contends 'using the SOC concept for making healthcare structure and culture as far as possible consistent, underload-overload balanced and participatory for patients, staff, and visitors would be an adequate and welcome application to make healthcare systems more salutogenic, generally'.

\section{Focus on the Production of Health and Encourage Movement towards the Health End of the Health ease/Dis-ease Continuum}

Antonovsky (1996, p. 14) explains, 'A salutogenic orientation, then, as the basis for health promotion, directs both research and action efforts to encompass all persons, wherever they are on the (healthy/dis-ease) continuum, and to focus on salutary factors'. Pelikan (2017, p. 262) adds that 'applying salutogenesis in health care 
successfully cannot just be done by introducing salutogenesis in healthcare practice, but must also include salutogenic clinical research, and change in underlying healthcare policy'. Rather than being limited to disease prevention and curative treatment, a salutogenic system prioritizes the creation and promotion of health in both research and policy. This is because the goal of institutionalized salutogenic health care is to move individuals and groups, wherever they may be situated on the health ease/dis-ease continuum towards the health end. As Lindström and Eriksson explain, (2010, p. 36) 'Salutogenesis again addresses mechanisms that enable people and populations to develop their health and deal with the fragmentation and chaos of reality through their senses of cognitive and emotional perception, behavioural skills and motivation through meaningful frameworks based on culture, tradition and belief systems'. Adopting Antonovsky's continuum model can help find effective ways to direct the increasing complexity of the healthcare system, reduce demands on the formal healthcare system, and foster the health-protective benefits of informal care. Lindström (2010, p. 10) argues 'future healthcare systems should focus much more on the mobilization of health resources and capabilities of patients in community settings and within the health institutions than previously done in the traditional approach'.

\section{Person-Centred and Integrative}

The idea of Person-Centered Medicine dates back to an emphasis on holism characteristic of the medical culture of Ancient Greece and has recently become resurgent, partly in response to limitations of sick care (Armstrong 2011; Snaedal 2012). At a basic level, Person-Centred Medicine is about recognizing and respecting the importance of each person as a unique and special case. Explaining how the model of care proffered by integrative medicine can inform salutogenic systems, Alivia et al. (2011, p. 382) note, 'Person-centred medicine takes into account the physical, psychological and spiritual aspects of a person in health and illness in order to individualise health promotion practices, diagnosis and treatment. It broadens the technological advances of Biomedicine with the epistemological approach, the relationship-based care and the salutogenetic treatments of non-conventional Medicine.' They contend, 'Such person-centred medicine aims to strengthen Antonovsky's concepts of resilience and sense of coherence with each therapeutic intervention so that overcoming illness becomes the foundation for better future health' (2011, p. 381). To develop a strong SOC people must feel socially valued. As such, with Person-Centered Medicine, the multi-dimensional uniqueness of each person and the complexity of their state of health is paramount. The main rationale is to 'take into account the totality of the person's health, and the idea that the person's aspirations and hopes, as well as his strengths and weaknesses, should be respected in an empowering collaboration between the person and those providing his medical care' (Snaedal 2012, p. 1 sic). This empowerment of the individual is accomplished in both health promotion activities and in medical practice 'through the practice of a clinical communication based on the salutogenic approach' (Lindström and Eriksson 
2010, p. 34). The perspective of the person receiving medical care or the object of health promotion is a focus of the salutogenic health care system.

\section{IIIness as Unavoidable and an Opportunity for Successful Adaptation}

In our medicocentric society the cultural script we are provided for understanding and dealing with illness is to view it as a personal tragedy; to be avoided at all costs through regimen and control and, failing that, to be fought in a heroic and high-tech medical battle, which ends up objectifying patients (Freund et al. 2003). Contrary to this, 'Antonovsky's research shows how adverse events and stress can become the opportunity to generate health if certain personal characteristics are present' (Alivia et al. 2011, p. 381). This means that a salutogenic health care system is one in which illness is regarded as an unpleasant but necessary precursor for a higher state of wellbeing. Analogous to the way in which microbial infection at first compromises and ultimately strengthens the immune system, health represents the ability to adapt to changing circumstances and environments. Alivia and colleagues (2011, p. 382) point out that 'A salutogenetic healthcare system needs to be oriented in such a way that illness is considered an adverse event that can become the foundation for better future health'. In a salutogenic orientation, illness is regarded as both unavoidable and as an important opportunity for successful adaptation through the ongoing pursuit of health and healing.

\section{Medical Security}

The ready availability of restorative medical care when we, or our loved ones, become sick or injured and need it is an important basis for having a well-developed SOC. The 'ontological security' (Giddens 1991) that there exists competent and caring medical professionals, supported by a well-resourced and institutionalized care system, able to respond to our sickness in a timely, appropriate, and effective manner is entirely salutogenic. The SOC depends on knowing that when we are sick or injured, there are people and places we can turn to for help. Indeed, the population health literature has for decades recognized appropriate and accessible formal healthcare as a major health determinant (Lalonde 1974; Epp 1986). This makes sense given the potential threat the experience of disease or injury poses to overwhelming our individual coping resources. Who among us is not thankful for the healing resources available and often taken-for-granted in developed countries, should we, as illustration, have the misfortune to be in a serious automobile accident? The list of available healing resources is astonishingly long and the takenfor-grantedness of these formal health care services reflects the magnitude of the contribution this form of medical security makes to the SOC. We can rest easy knowing that when the unthinkable happens, we will be taken care of. The result of medical security, then, is to make you feel as though you have been well cared for. Antonovsky (1979, p. 217) puts it most cogently: 'A society, then, that has institutionalized a healthcare system that expresses consensus that healthcare is an inalienable right of all its citizens and is to be made equally available to all on the 
universalistic ground of being a resident of that society is a society that has taken a step forward in strengthening the sense of coherence of its members'.

Of course, different societies have different mechanisms for achieving this sort of medical security, if at all. Health care systems are reflective of the cultural and ideological features of the societies which give rise to them and in which they function. Antonovsky (1979, p. 199) was acutely aware of this writing, 'If, indeed many of the pressures impinging on a given population group are in the direction of forming a weak sense of coherence, then it is likely that its members' experiences with healthcare workers and the healthcare institution will point in the same direction'. By this he means that sick societies allow sick care systems to flourish by undermining the existential security afforded through medical security. One thinks immediately of the situation in the United States and the collapsing of The Patient Protection and Affordable Care Act (ACA; Obamacare).

\section{Conclusion}

Pelikan (2017, p. 261) advises 'in principle applying salutogenesis to healthcare means to restrict the leading pathogenic orientation in healthcare practice (research and policy) and complement or change it by a salutogenic orientation in every day practice and research'. This is an ambitious vision to be sure. Further, the current political climate, characterized by neoliberal domination, means that there are political, economic, and sociocultural factors that stand as barriers that must be overcome to achieve the vision of healing healthcare in policy and in clinical practice. Politically, deepening social and health inequalities are embedded within the structural organization of neoliberal governance. In such a political context, formal health care is further limited in the already small contribution it can make to population health. Chief among economic barriers is that medical dominance has mutated into a widespread medicalization of life that has paradoxically expanded the influence of the medical-industrial complex in health and healing, while undermining the professional autonomy of organized medicine in the face of powerful vested interests such as the biotechnological and pharmaceutical industries. These political and economic barriers are compounded by sociocultural factors in which the pathogenic orientation of contemporary formal health care is deeply embedded within the culture of the developed West. The present medicocentric culture is characterized by widespread confusion between 'medicine' and 'health', with the general public and health care experts alike mistaking the consumption of formal healthcare services for the production of population health (Evans and Stoddart 1990). This allows the medical-industrial complex to profit from trading in sickness and pathogenesis, while circumscribing political discourse to debates concerning the provision of sick care. Barriers such as these have diminished the adaptive capacity of the healthcare system.

The nested complexity of health means that health and healthcare policies must above all else be flexible in the face of changing circumstances of population health. As Geyer and Rihani (2010, p. 32) argue, 'the key is not to find the final order and implement it, but encourage the actors in the policy arena to adapt and adjust to the 
continual evolutionary changes'. The goal is to move the healthcare system into 'the zone of creative complexity' while avoiding 'disorder and stifling order' (Geyer and Rihani 2010, p. 36). Moving from sick care towards salutogenic healing systems to enhance health as adaptive capacity means enacting both a cultural shift challenging the medical dominance that has produced the contemporary medicalized care system and fundamental social change directed towards overcoming existing political, economic, and sociocultural barriers that stand in the way of population health. Professional medicine and medical research will both have to reoriente to support population health promotion as engines of creative complexity.

A central objective of this analysis has been to provide a conceptual basis needed to heal the healthcare system by translating salutogenic insights into bases for restructuring the formal healthcare system. As Lindström and Eriksson argue, (2010, p. 36) 'the potential of the salutogenic concept lies in its implications for creating societies that adopt a healthy public policy, where the content and the structure of all services are salutogenic, rather than a healthy policy only for the health services'. As insights from complexity based analyses of health make clear, all policies affect health and well-being and therefore, viewed through a salutogenic perspective, all policies need to be reconceived as health policy; not just those pertaining to the provision of formal healthcare. Barriers against moving from sick care towards salutogenic healing systems are political, economic, and sociocultural. Just as health is multidimensional so too must healthcare policies and systems operate within the zone of creative complexity. The health care system must adapt to demographic trends, such as population aging and the epidemiological transition from infectious to chronic disease, in order to meet emerging healthcare needs such as prevention, long term chronic care, and end of life care. Ultimately, healing the health care system depends on looking at health through a salutogenic perspective and a societal shift that reallocates resources to move beyond trying to repair the current sickness care system and create a salutogenic health care system that contributes to the production of population health and wellness. Kickbusch (2007, p. 147) nicely articulates the rationale motivating such a challenge: 'Health is considered a right and its do-ability is driven not only by universal access to the medical health system but also by the salutogenic promise that health can be created, managed and produced by addressing the determinants of health as well as by influencing behavior and lifestyles'. The salutogenic model is valuable because it directs focus to the realization that improvement of the healthcare system rests upon understanding population health as a complex nested system (Antonovsky 1979, 1987). For a healthcare system to be salutogenic, it must adopt a complex focus on groups and the social determinants of health (Antonovsky 1979). The development of effective healthcare requires a thorough understanding of the complex, overlapping factors, which make people healthy. Unfortunately, sick care and so-called 'health research' have primarily continued a mechanistic focus on pathogenesis and disease prevention. Medical care and research continue to be constrained by a pathogenic orientation. Antonovsky's salutogenic theory offers an alternative, less medicocentric vision of future health care policy and programs. His continuum model offers a productive basis for analysing health and healthcare systems. It allows us to grasp that debates between population health promotion and providing medical care, are, at their root, 
unproductive debates predicated on a false dichotomy. A truly salutogenic health care system is one which pays credence to the multidimensional complexity of human health and strives to strike an adaptive balance between health production and the provision of medical care. This makes sense if we realize that we are all, as Antonovsky (1987, p. 3) describes, 'terminal cases' just as 'we are all, so long as there is a breath of life in us, in some measure healthy'. A salutogenic healthcare system is one which rests upon understanding the unitary ontology of health and sickness that lays at the heart of Antonovsky's mystery of health.

\section{References}

Alivia, M., P. Guadagni, and P.R. di Sarsina. 2011. Towards salutogenesis in the development of personalised and preventive healthcare. European Association for Predictive, Preventive and Personalised Medicine 2: 381-384.

Antonovsky, A. 1979. Health, stress, and coping. San Francisco: Jossey-Bass Publishers.

Antonovsky, Aaron. 1987. Unravelling the mystery of health. San Francisco: Jossey-Bass.

Antonovsky, Aaron. 1996. The salutogenic model as a theory to guide health promotion. Health Promotion International 11 (1): 11-18.

Armstrong, D. 2011. The invention of patient-centred medicine. Social Theory \& Health 9 (4): 410-418.

Ayo, N. 2012. Understanding health promotion in a neoliberal climate and the making of health conscious citizens. Critical Public Health 22: 99-105.

Ballard, K., and M. Elston. 2005. Medicalisation: A multi-dimensional concept. Social Theory and Health 3: 228-241.

Becker, C. M., M. A. Glascoff, W. M. Felts. 2010. Salutogenesis 30 years later: Where do we go from here? International Electronic Journal of Health Education 13: 25-32.

Bourdieu, P. 1977. Outline of a theory of practice. Palo Alto, CA: Stanford University Press.

Breslow, L. 2004. The third revolution in health. Annual Review of Public Health 25 (1): xiii-xviii.

Breslow, L. (1972). A quantitative approach to the World Health Organization definition of health: physical, mental and social well-being. International journal of Epidemiology, 1(4), 347-355

Busfield, J. 2017. The concept of medicalisation reassessed. Sociology of Health \& Illness 39 (5): 759-774.

Canadian Institute for Health Information. 2015. National health expenditure trends, 1975 to 2015. Ottawa: Canadian Institute for Health Information.

Conrad, P. 2007. The medicalization of society. Baltimore, MD: Johns Hopkins University Press.

Epp, J. 1986. Achieving health for all: A framework for health promotion. Ottawa: Health and Welfare Canada.

Eriksson, M., and B. Lindstrom. 2008. A salutogenic interpretation of the Ottawa Charter. Health Promotion International 23: 190-199.

Eriksson, M., and M.B. Mittelmark. 2017. The sense of coherence and its measurement. In The handbook of salutogenesis, ed. M.B. Mittelmark et al., 97-106. New York: Springer.

Evans, R.G., and G.L. Stoddart. 1990. Producing health, consuming health care. Social Science and Medicine 31 (12): 1347-1363.

Freidson, E. 1970a. Professional dominance: The social structure of medical care. New York: Atherton.

Freidson, E. 1970b. Profession of medicine: A study of the sociology of applied knowledge. New York: Harper and Row.

Freund, P., E.S. Meridith, B. McGuire, and L.S. Podhurst. 2003. Health, illness, and the social body: A critical sociology. New York: Prentice Hall.

Friedli, L. 2013. What we've tried, hasn't worked': The politics of assets based public health. Critical Public Health 23 (2): 131-145.

Fries, C.J. 2008. Governing the health of the hybrid self: Integrative medicine, neoliberalism, and the shifting biopolitics of subjectivity. Health Sociology Review 17: 353-367.

Geyer, R., and S. Rihani. 2010. Complexity and public policy: A new approach to twenty-first century politics, policy and society. London: Routledge. 
Giddens, A. 1991. Modernity and self identity: Self and society in late modern age. Cambridge: Polity.

Henly, S.J., J.F. Wyman, and M.J. Findorff. 2011. Health and illness over time: The trajectory perspective in nursing science. Nursing Research 60 (3 Suppl): S5.

Holman, D., R. Lynch, and A. Reeves. 2018. How do health behaviour interventions take account of social context? A literature trend and co-citation analysis. Health 22 (4): 389-410.

Illich, I. 1976. Medical nemesis: The expropriation of health. New York: Pantheon Books.

Jonas, W.B., R.A. Chez, K. Smith, and B. Sakallaris. 2014. Salutogenesis: The defining concept for a new healthcare system. Global Advances in Health and Medicine 3 (3): 82-91.

Kickbusch, I. 2007. Health governance: The health society. In Health and modernity: The role of theory in health promotion, ed. D. McQueen et al., 144-161. New York: Springer.

Lalonde, M. 1974. A new perspective on the health of Canadians. Ottawa: Health and Welfare Canada.

Lindström, B. 2010. Salutogenesis—an introduction. Retrieved from http://www.ndphs.org/docum ents/2502/SALUTOGEN\%20ESIS\%20and\%20NCDs.pdf.

Lindström, B., and M. Eriksson. 2010. A salutogenic approach to tackling health inequalities. In Health assets in a global context: Theory, methods, action, ed. A. Morgan et al., 17-39. New York: Springer.

Lupton, D. 1995. The imperative of health: Public health and the regulated body. London: Sage.

Markle, G.E., and F.B. McCrea. 2008. What if medicine disappeared?. Albany, NY: SUNY Press.

Millar, J., and C. Hull. 1997. Measuring human wellness. Social Indicators Research 40: 147-158.

Morgan, M., M. Davies, and E. Ziglio (eds.). 2010. Health assets in a global context: Theory, methods, action. New York: Springer.

Naylor, C.D. 2003. Learning from SARS: Renewal of Public Health in Canada: A Report of the National Advisory Committee on SARS and Public Health. Ottawa: National Advisory Committee.

OECD. 2017. Health at a glance 2017: OECD indicators. Paris: OECD. https://doi.org/10.1787/healt h_glance-2017-en.

Pelikan, J.M. 2017. The application of salutogenesis in healthcare settings. In The handbook of salutogenesis, ed. M.B. Mittelmark et al., 261-266. New York: Springer.

Renaud, M. 1994. The future: Hygeia versus Panakeia. In Why are some people healthy and others not?, ed. R.G. Evans et al., 317-334. New York: Aldine De Gruyter.

Rose, G., K.T. Khaw, and M. Marmot. 2008. Rose's strategy of preventive medicine: The complete original text. New York: Oxford University Press.

Schrecker, T., and C. Bambra. 2015. How politics makes us sick: Neoliberal epidemics. Berlin: Springer.

Segall, A., and C.J. Fries. 2017. Pursuing health and wellness: Healthy societies, healthy people, $2 \mathrm{nd}$ ed. Toronto: Oxford University Press.

Snaedal, J. 2012. Person centered medicine. World Medical \& Health Policy 4 (2): 1-14.

Tones, K., and J. Green. 2004. Health promotion: Planning and strategies. London: Sage.

Van den Bogaert, S., R.A. Ayala, and P. Bracke. 2017. Beyond ubiquity: Unravelling medicalisation within the frame of health insurance and health-policy making. Social Theory \& Health 15 (4): 407-429.

Zola, Irving K. 1972. Medicine as an institution of social control. The Sociological Review 20 (4): 487-504.

Publisher's Note Springer Nature remains neutral with regard to jurisdictional claims in published maps and institutional affiliations. 